\title{
Transverse vibrations of clamped and simply-supported circular plates with two dimensional thickness variation
}

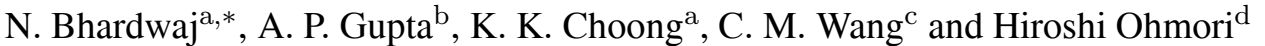 \\ ${ }^{a}$ School of Civil Engineering, Universiti Sains Malaysia, Engineering Campus, 14300 Nibong Tebal, Seberang \\ Perai Selatan, Pulau Pinang, Malaysia \\ ${ }^{\mathrm{b}}$ Department of Applied Sciences and Humanities, Huda-Sector 23-A, ITM University, Gurgaon-Haryana-122017, \\ India \\ ${ }^{c}$ Department of Civil Engineering, National University of Singapore, Kent Ridge, Singapore \\ ${ }^{\mathrm{d}}$ Department of Environmental Engineering and Architecture, School of Environmental Studies, Nagoya University, \\ Japan
}

Received 20 May 2008

Revised 23 September 2010

\begin{abstract}
Two dimensional boundary characteristic orthonormal polynomials are used in the Ritz method for the vibration analysis of clamped and simply-supported circular plates of varying thickness. The thickness variation in the radial direction is linear whereas in the circumferential direction the thickness varies according to $\cos k \theta$, where $k$ is an integer. In order to verify the validity, convergence and accuracy of the results, comparison studies are made against existing results for the special case of linearly tapered thickness plates. Variations in frequencies for the first six normal modes of vibration and mode shapes for various taper parameters are presented.
\end{abstract}

\section{Introduction}

Plates of various shapes and of non-uniform thickness are widely used in engineering structures. Many papers have been written on the vibration of plates covering various shapes, thickness variations according to different power laws and different boundary conditions. Leissa [1] have discussed an excellent series of survey papers in his book: vibration of plates. Focusing attention on plates vibration with varying thickness, Laura and Gutierrez [3] have analyzed the vibration of circular plates with non-uniform thickness by means of the differential quadrature method. Laura and his co-workers [4] have studied the problem of free vibration of a solid circular plate of linearly varying thickness resting on a Winkler foundation. In another paper Bambill and Laura [5] have solved axisymmetric vibration of circular plates with double linear variable thickness. Chakraverty et al. [6] have surveyed the research on vibration of plates using boundary characteristic polynomials in the Rayleigh-Ritz method. Forced axisymmetric and asymmetric responses of linearly tapered circular plates have been studied by Gupta and Goyal [7,8]. Kim and Dickinson [9] have investigated the lateral vibrations of thin annular and circular composite plates subjected to certain complicating effects. Chen [10] has analyzed axisymmetric vibrations of circular and annular plates with arbitrarily varying thickness. Wang [11] has studied classical circular plates with generalized variable thickness and presented power series solutions in the form of recursive relations whereas exact vibration results for stepped

\footnotetext{
*Corresponding author: Neeraj Bhardwaj, E-mail: bneerajdma@gmail.com.
} 


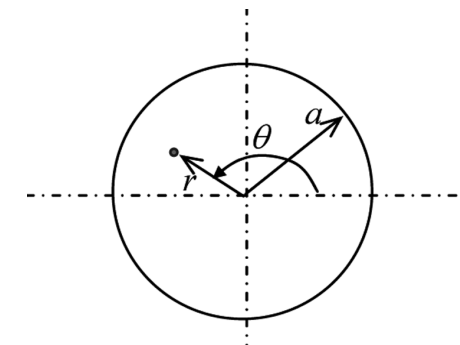

(a)

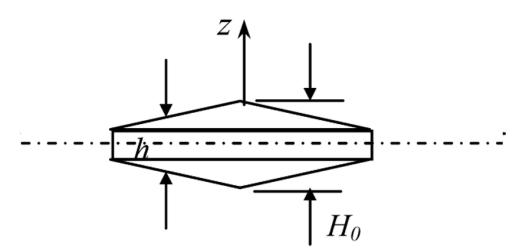

(b)

Fig. 1. Plate geometry: (a) plan view and (b) side view.

circular plates with free edges has been obtained by Hang et al. [12]. These vibration results are used for the hydro elastic analysis of circular large floating structures. Duan et al. [13] have treated an interesting problem of modifying the fundamental vibration modes of circular plates with free edges. In recent years, Bhardwaj and his co-workers [14-20] have analyzed different structural orthotropic tapered plate problems resting on Winkler elastic foundations using the Rayleigh-Ritz method. Ansari and Gupta [21] have solved the problem of forced vibration of axisymmetric polar orthotropic plates resting on elastic foundation. Civalek and Ozturk [22] have analyzed free vibration of tapered rectangular plates using the discrete singular convolution method. The same method has used by Civalek [23] to determine the fundamental frequency and to study the effect of mode number of isotropic and orthotropic rectangular plates with linearly varying thickness.

Hitherto, no study has been made on the vibrations of circular plates whose thickness varies linearly in the radial direction and according to $\cos k \theta$ in the circumferential (or $\theta$ ) direction, where $k$ is an integer. Such plate thickness variations find applications in body vibrating /massage machines and can be potentially used as rotating disk in devices to generate vortex flow in fluid or to generate optical vortices. The objective of this study is to investigate the vibration frequencies and the corresponding mode shapes for such varying thickness circular plates. For analysis, boundary characteristic orthonormal polynomials are used as admissible functions in the Ritz method. Four types of thickness variations are considered in $\theta$-direction by adjusting the integer $k$ (i.e., $k=1,2,3$ and 4 ). Frequencies for the first six normal modes of vibration for various values of taper parameters for clamped circular and simply-supported plates are computed and these results are presented in graphical forms. Contour plots of mode shapes are also presented. In order to verify the correctness of the converged results, comparison studies are made with particular cases that are available in the literature and shown in tabular form.

\section{Problem formulation and analysis}

Let us consider an isotropic, thin circular plate of radius $a$, and varying thickness $h(r, \theta)$ as shown in Fig. 1. The formulation is presented in cylindrical coordinates with the axis of the plate along the $\mathrm{z}$-axis and the middle plane of the plate in the $r-\theta$ plane.

The energy functional $J(W)$ is obtained by subtracting the maximum kinetic energy from the maximum strain energy, i.e.

$$
\begin{aligned}
J(W)= & \frac{E H_{0}^{3}}{24\left(1-\nu^{2}\right)} \int_{0}^{1} \int_{0}^{2 \pi}\left[F ^ { 3 } ( R , \theta ) \left\{W,_{R R}^{2}+2 \nu W, R R\left(\frac{1}{R^{2}} W,_{\theta \theta}+\frac{1}{R} W,_{R}\right)+\left(\frac{1}{R^{2}} W,_{\theta \theta}+\frac{1}{R} W,_{R}\right)^{2}\right.\right. \\
& \left.\left.+4\left(1-\nu^{2}\right)\left(\frac{1}{R^{2}} W,_{\theta}-\frac{1}{R} W, R \theta\right)^{2}\right\}-\Omega^{2} F W^{2}\right] R d \theta d R, \quad \ldots
\end{aligned}
$$

where $H=h / a, \quad R=r / a, w(r, \theta, t)=a W(R, \theta) \cos \omega T, T=(t / a) \sqrt{E / \rho}, \Omega^{2}=12\left(1-\nu^{2}\right) \omega^{2} / H_{0}^{2}$, $H_{0}$ is the thickness at the centre of plate, $\rho$ the mass density, $w(r, \theta, t)$ the transverse deflection and $\omega$ the natural 

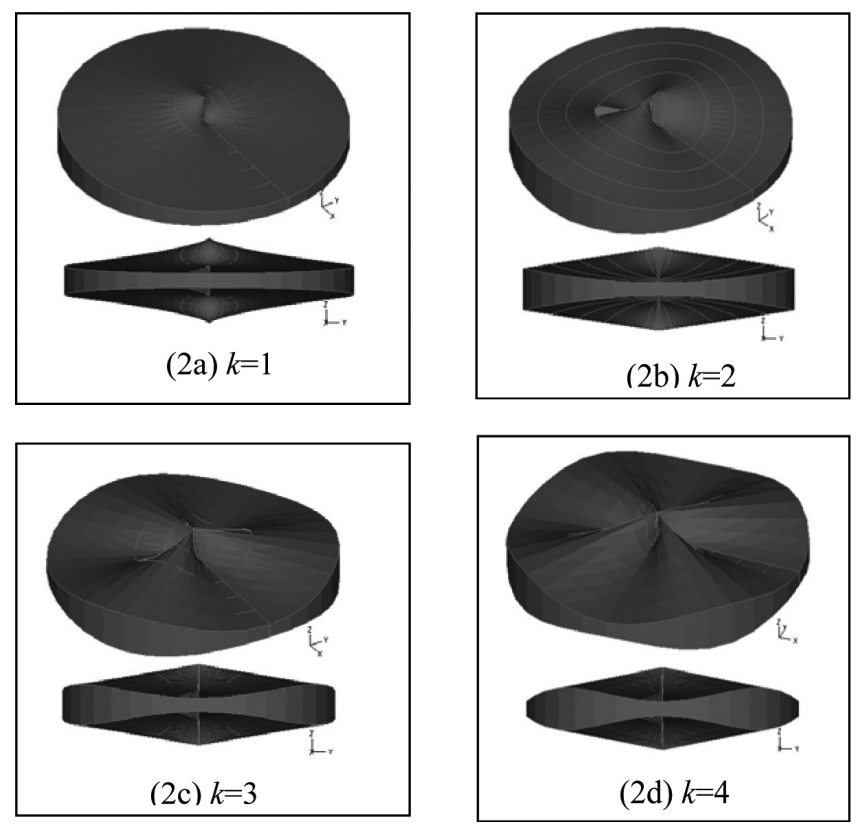

Fig. 2. Three dimensional view of plate geometry when $k=1$ to 4 .

circular frequency of the plate. A comma followed by a suffixed variable denotes differentiation with respect to that variable.

The considered two dimensional variation in thickness is taken as

$$
H=H_{0} F(R, \theta) \text {, where } F(R, \theta)=(1-\alpha R)(1-\beta \cos k \theta), \ldots
$$

where $k$ is an integer and $\alpha, \beta$ are the taper constants with assumption that $\beta=0$ at $r=0$. The plate thickness variations considered here are shown in Figs 2(a) to 2(d) where the four cases show the changes in plate geometry as the parameter $k$, varies from 1 to 4 . In all cases of plate geometry, both taper parameters $\alpha$ and $\beta$ are assigned positive values. The assignment of negative $\alpha$ (with $\beta=0$ ) will lead to plate tapering in decreasing thickness along radial direction towards edge of plate. On the other hand, the assignment of negative $\beta$ (with $\alpha=0$ ) will lead to changes in pattern of thickness variation in circumferential direction from ridge to valley and vice-versa in comparison to the case of positive $\beta$.

According to the Ritz method, the functional $J(W)$ is to be minimized with respect to $W$. The deflection $W$ may be approximated by

$$
W(R, \theta)=\sum_{j=1}^{N} c_{j} \Phi_{j}(R, \theta), \quad \ldots
$$

where $\Phi_{j}(R, \theta)$ are the two dimensional boundary characteristic orthonormal polynomials and they are generated by the Gram-Schmidt process as follows:

$$
\begin{aligned}
& \Phi_{j}=\phi_{j} / \sqrt{<\phi_{j}, \phi_{j}>}, \quad \phi_{1}=\left(1-R^{2}\right)^{p} \quad \ldots \\
& \phi_{i}=\phi_{1} f_{i}(R, \theta)-\sum_{j=1}^{i-1} \frac{<\phi_{1} f_{i}, \phi_{j}>}{<\phi_{j}, \phi_{j}>} \phi_{j}, \quad i=2(1) N, \quad \ldots \\
& <f, g>=\iint_{A} F(R, \theta) f(R, \theta) g(R, \theta) R d \theta d R, \quad \ldots
\end{aligned}
$$

where $A$ is the area of the plate. 
The functions $f_{i}(R, \theta)$ are taken as simple polynomials of $R \cos \theta$ and $R \sin \theta$ of the form $R^{m_{i}+n_{i}} \cos ^{m_{i}} \theta$ $\sin ^{n_{i}} \theta$, where $m_{i}$ and $n_{i}$ are non-negative integers taken as:

$\begin{array}{crrrrrrrrrrrrrrrl}i & 1 & 2 & 3 & 4 & 5 & 6 & 7 & 8 & 9 & 10 & 11 & 12 & 13 & 14 & 15 & \ldots \\ m_{i} & 0 & 1 & 0 & 2 & 1 & 0 & 3 & 2 & 1 & 0 & 4 & 3 & 2 & 1 & 0 & \ldots \\ n_{i} & 0 & 0 & 1 & 0 & 1 & 2 & 0 & 1 & 2 & 3 & 0 & 1 & 2 & 3 & 4 & \ldots\end{array}$

The substitution of $W(R, \theta)$ from Eq. (3) into the energy functional [Eq. (1)] and then minimizing $J(W)$ as a function of the coefficients $c_{j}$ leads to the standard eigenvalue problem:

$$
\sum_{j=1}^{N}\left(a_{i j}-\Omega^{2} \delta_{i j}\right) c_{j}=0, \quad i=1(1) \quad N, \quad \ldots
$$

where

$$
\delta_{i j}=\left\{\begin{array}{l}
1 \text { when } i=j \\
0 \text { when } i \neq j
\end{array}\right.
$$

and

$$
\begin{aligned}
a_{i j}= & \int_{0}^{1} \int_{0}^{2 \pi}\left[F ^ { 3 } ( R , \theta ) \left\{\Phi_{i, R R} \Phi_{j, R R}+\nu \Phi_{i, R R}\left(\frac{1}{R^{2}} \Phi_{j, \theta \theta}+\frac{1}{R} \Phi_{j, R}\right)+\nu \Phi_{j, R R}\left(\frac{1}{R^{2}} \Phi_{i, \theta \theta}+\frac{1}{R} \Phi_{i, R}\right)\right.\right. \\
& +\left(\frac{1}{R^{2}} \Phi_{i, \theta \theta}+\frac{1}{R} \Phi_{i, R}\right)\left(\frac{1}{R^{2}} \Phi_{j, \theta \theta}+\frac{1}{R} \Phi_{j, R}\right)+4\left(1-\nu^{2}\right)\left(\frac{1}{R^{2}} \Phi_{i, R \theta}-\frac{1}{R} \Phi_{i, \theta}\right) \\
& \left.\left.\left(\frac{1}{R} \Phi_{j, R \theta}-\frac{1}{R^{2}} \Phi_{j, \theta}\right)\right\}+\Phi_{i} \Phi_{j}\right] R d \theta d R
\end{aligned}
$$

The integral evaluated in Eqs (6) and (8) are given by the formula

$$
\begin{aligned}
\int_{0}^{1} \int_{0}^{2 \pi} & \left(1-R^{2}\right)^{i} R^{j+l} \cos ^{j} \theta \sin ^{l} \theta R d \theta d R \\
& =\sqrt{\frac{i+1}{2}} \sqrt{\frac{j+l+2}{2}} \sqrt{\frac{j+1}{2}} \sqrt{\frac{l+1}{2}} / \sqrt{\frac{2 i+j+l+4}{2}} \sqrt{\frac{j+l+2}{2}} \text { when } j \text { and } l \text { are even, } \\
= & 0, \text { otherwise, ... }
\end{aligned}
$$

where $i, j, l>-1$.

The eigenvalues $(\Omega)$ and the eigenvectors $\left(c_{j}\right)$ are computed by the Jacobi method. The mode shapes are computed from Eq. (3) and their corresponding contours are plotted. $\mathrm{C}++$ coding is employed for all the computations.

\section{Results and discussion}

There are six parameters (i.e., $\alpha, \beta, k, p, N$ and $\nu$ ) used in the analysis of the plate. The value of $\nu$ is taken as 0.3 for all calculations except for calculating the results given in Table 3 where $\nu$ is taken as 0.33 for the sake of the comparison with known results. The value of parameter $p$ is taken as 2 or 1 for clamped edge or simply-supported edge. The taper parameters $\alpha$ and $\beta$ vary from -0.9 to 0.9 and -0.7 to 0.7 , respectively, with a step size of 0.2 . Four cases of thickness variations corresponding to $k=1,2,3$ and 4 are considered. Each case is discussed separately.

In Table 1, the convergence for first six normal modes of vibrations at least up to five significant figures for the clamped (C-plate) and simply-supported plates (S-plate) are investigated for $\alpha=\beta=0.5$. It is observed that a maximum of 19 terms are required to obtain converged results in all four cases, i.e., $k=1,2,3,4$. It is observed that the frequencies of $\mathbf{C}$-plates are more than that of $\mathbf{S}$-plates as expected due to the higher stiffness of the $\mathbf{C}$-plates in comparison to $\mathbf{S}$-plates for all the four cases of circumferential thickness variations. Moreover, there is no specific 
Table 1

Convergence of $\Omega$ for $\mathrm{C}$ and $S$ - plates when $\alpha=\beta=0.5, e_{r}=1.0, g_{r}=0.384$

\begin{tabular}{|c|c|c|c|c|c|c|c|c|c|c|}
\hline \multirow{2}{*}{$\begin{array}{c}\text { Case-I } \\
\rightarrow \Omega^{N}\end{array}$} & \multicolumn{5}{|c|}{ C-plate } & \multicolumn{5}{|c|}{ S-plate } \\
\hline & 15 & 16 & 17 & 18 & 19 & 15 & 16 & 17 & 18 & 19 \\
\hline$\Omega_{1}$ & 5.1345 & 5.1282 & 5.1282 & 5.1280 & 5.1280 & 2.8402 & 2.8265 & 2.8263 & 2.8261 & 2.8261 \\
\hline$\Omega_{2}$ & 11.424 & 11.424 & 11.423 & 11.423 & 11.423 & 8.1840 & 8.1840 & 8.0850 & 8.0850 & 8.0850 \\
\hline$\Omega_{3}$ & 12.203 & 12.200 & 12.200 & 12.149 & 12.149 & 9.1957 & 8.8380 & 8.8380 & 8.7684 & 8.7684 \\
\hline$\Omega_{4}$ & 18.991 & 18.991 & 18.741 & 18.741 & 18.571 & 15.587 & 15.488 & 15.350 & 15.350 & 15.350 \\
\hline$\Omega_{5}$ & 19.288 & 19.098 & 19.098 & 18.930 & 18.930 & 15.620 & 15.587 & 15.488 & 15.487 & 15.487 \\
\hline$\Omega_{6}$ & 23.463 & 23.110 & 23.110 & 23.110 & 23.110 & 21.095 & 20.784 & 20.780 & 20.321 & 20.321 \\
\hline Case-II & & & C-plate & & & & & S-plate & & \\
\hline$\Omega_{1}$ & 5.8316 & 5.8316 & 5.8316 & 5.8316 & 5.8316 & 3.4113 & 3.4113 & 3.4113 & 3.4113 & 3.4113 \\
\hline$\Omega_{2}$ & 9.8361 & 9.8352 & 9.8352 & 9.7849 & 9.7849 & 9.2911 & 8.9958 & 8.8186 & 8.8186 & 8.8186 \\
\hline$\Omega_{3}$ & 15.497 & 15.497 & 15.494 & 15.494 & 15.286 & 11.376 & 11.376 & 11.270 & 11.270 & 11.270 \\
\hline$\Omega_{4}$ & 19.681 & 19.681 & 19.681 & 19.681 & 19.681 & 18.308 & 18.308 & 18.308 & 18.308 & 18.308 \\
\hline$\Omega_{5}$ & 19.807 & 19.807 & 19.807 & 19.807 & 19.807 & 18.718 & 18.718 & 18.718 & 18.718 & 18.718 \\
\hline$\Omega_{6}$ & 29.471 & 28.534 & 28.534 & 26.136 & 26.136 & 24.291 & 24.291 & 24.291 & 24.291 & 24.291 \\
\hline Case-III & & & C-plate & & & & & S-plate & & \\
\hline$\Omega_{1}$ & 6.3833 & 6.3515 & 6.3515 & 6.3251 & 6.3251 & 3.7980 & 3.7320 & 3.7008 & 3.7008 & 3.7008 \\
\hline$\Omega_{2}$ & 12.464 & 12.434 & 12.427 & 12.322 & 12.322 & 10.345 & 10.331 & 10.258 & 10.258 & 10.258 \\
\hline$\Omega_{3}$ & 23.848 & 23.848 & 23.772 & 22.347 & 22.347 & 10.364 & 10.346 & 10.331 & 10.310 & 10.310 \\
\hline$\Omega_{4}$ & 30.230 & 29.637 & 29.637 & 29.310 & 29.310 & 20.249 & 20.211 & 19.592 & 19.592 & 19.397 \\
\hline$\Omega_{5}$ & 32.585 & 32.585 & 32.520 & 32.520 & 32.520 & 20.249 & 20.249 & 20.210 & 19.935 & 19.592 \\
\hline$\Omega_{6}$ & 37.003 & 36.998 & 36.996 & 36.994 & 36.994 & 24.243 & 24.230 & 24.228 & 24.226 & 24.226 \\
\hline Case-IV & & & C-plate & & & & & S-plate & & \\
\hline$\Omega_{1}$ & 6.6497 & 6.6497 & 6.6497 & 6.6497 & 6.6497 & 3.8259 & 3.8259 & 3.8259 & 3.8259 & 3.8259 \\
\hline$\Omega_{2}$ & 13.773 & 13.369 & 13.414 & 13.414 & 13.162 & 9.7779 & 9.6897 & 9.3353 & 9.3353 & 9.2001 \\
\hline$\Omega_{3}$ & 13.773 & 13.773 & 13.769 & 13.630 & 13.630 & 9.7779 & 9.6897 & 9.6897 & 9.6326 & 9.6326 \\
\hline$\Omega_{4}$ & 18.162 & 18.162 & 18.162 & 18.162 & 18.162 & 13.486 & 13.486 & 13.486 & 13.486 & 13.486 \\
\hline$\Omega_{5}$ & 27.267 & 27.267 & 27.267 & 27.267 & 27.267 & 20.248 & 20.248 & 20.248 & 20.248 & 20.248 \\
\hline$\Omega_{6}$ & 30.753 & 30.753 & 30.753 & 30.753 & 30.753 & 24.245 & 24.245 & 24.245 & 24.245 & 24.245 \\
\hline
\end{tabular}

Table 2

Comparison of $\Omega$ for isotropic $C$ and $S$ - plates when $\alpha=\beta=0$ and $e_{r}=1$

\begin{tabular}{ccccccccc}
\hline $\begin{array}{c}\text { Edge } \\
\text { Condition }\end{array}$ & Ref. & $\Omega_{1}$ & $\Omega_{2}$ & $\Omega_{3}$ & $\Omega_{4}$ & $\Omega_{5}$ & $\Omega_{6}$ & $\Omega_{7}$ \\
\hline$S$ & {$[9]$} & 4.93515 & 13.8982 & 25.6133 & 29.720 & 39.954 & 48.4796 & 56.8425 \\
& Present & 4.93515 & 13.896 & 25.6129 & 29.720 & 39.954 & 48.477 & 56.841 \\
\multirow{2}{*}{$C$} & {$[9]$} & 10.2158 & 21.260 & 34.877 & 39.771 & 51.030 & 60.829 & 69.674 \\
& Present & 10.216 & 21.258 & 34.872 & 39.771 & 51.025 & 60.827 & 69.666 \\
\hline
\end{tabular}

pattern of variation of the frequencies is found for both $\mathbf{C}$ - and $\mathbf{S}$-plates except that the first mode shows an increase in frequencies with an increase in $k$ (from 1 to 4 ).

A comparison of the frequency parameters $\Omega$ with Kim and Dickinson [9] results for isotropic simply-supported and clamped plates of uniform thickness $(\alpha=\beta=0)$ is shown in Table 2 with $\nu=0.3$. In Table 3 comparison of $\Omega$ is shown with the results of Gelos [24], Luisoni [25], Wah [26], Gontkevich [27], Avolos [28], Leissa and Narita [2] and Leissa [1] for isotropic S-plates of uniform thickness when $e_{r}=1.0$ and $\nu=0.3$.

Comparison of $\Omega$ with Leissa and Narita [2], Singh and Saxena [29] and Laura and his co-worker's [4] for isotropic S-plates of linearly varying (L.V.) thickness ( $\alpha$ varying and $\beta=0)$ when $e_{r}=1.0$ and $\nu=0.3$ is shown in Table 4 and for the same parameters as C-plates with the results of Laura and his co-worker's [4] given in Table 5. In Tables 2 to 5, the maximum quantitative difference in frequencies relative to the existing results is found $\pm 0.84 \%$.

The variations of the first six frequencies with respect to the taper parameters $\alpha$ and $\beta$ for $k=1,2,3$ and 4 are shown in Figs 3 and 4 . Figure 3 shows the variation of $\Omega$ for $\alpha$ ranging from -0.7 to 0.7 while $\beta$ is kept constant (i.e. $\beta=0.7$ ). It can be seen that all frequencies decrease with increasing taper parameter $\alpha$. This is expected since varying $\alpha$ from negative to positive means that the mass of the plates (in all the four cases $k=1,2,3$ and 4) will be shifted from around the supporting edges towards the plate centre due to the larger thickness at the center, resulting 


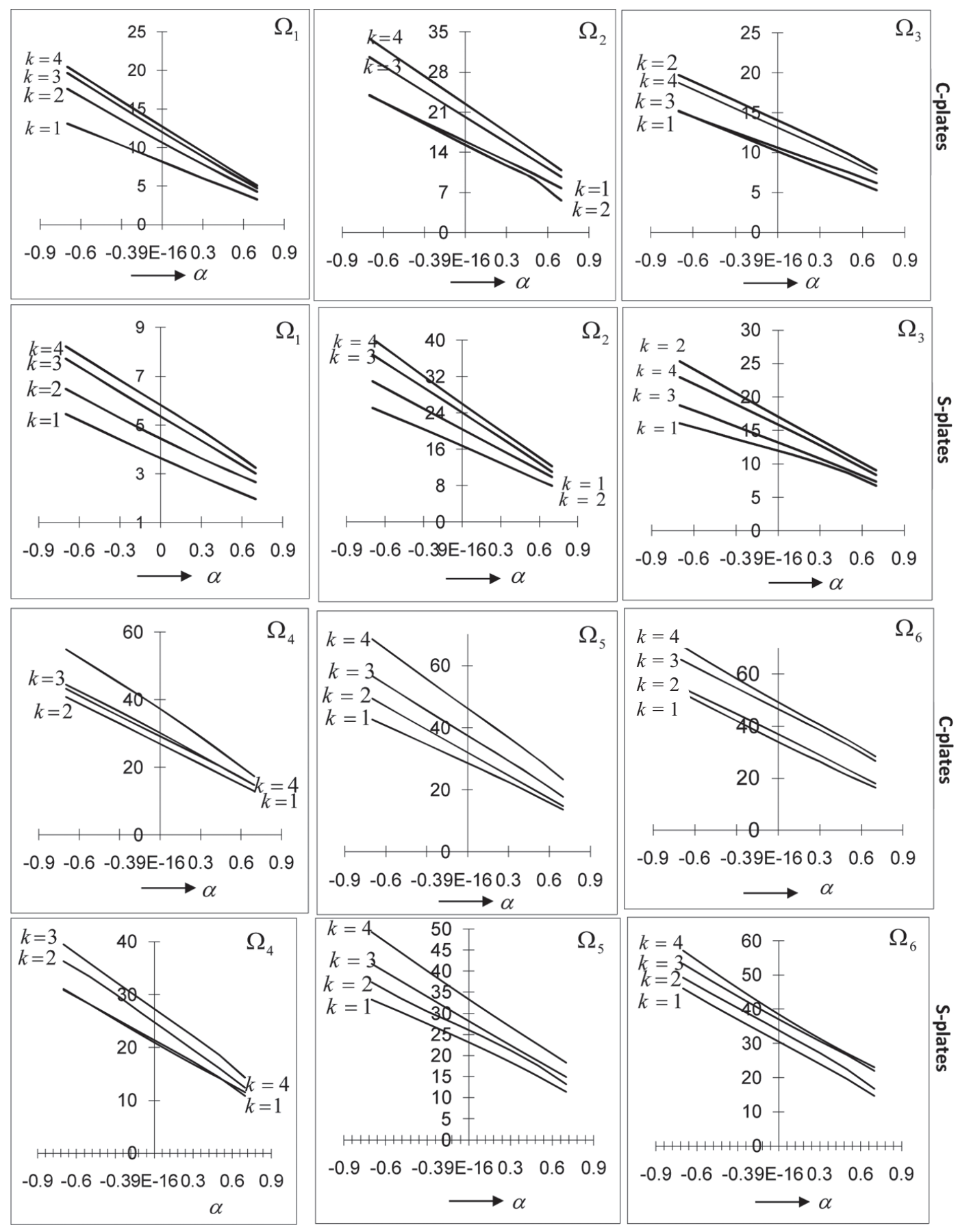

Fig. 3. Variation in $\Omega$ with $\alpha$ for corresponding $\mathrm{C}$ and S- plates when $\beta=0.7$ and $k=1,2,3$ and 4 . 

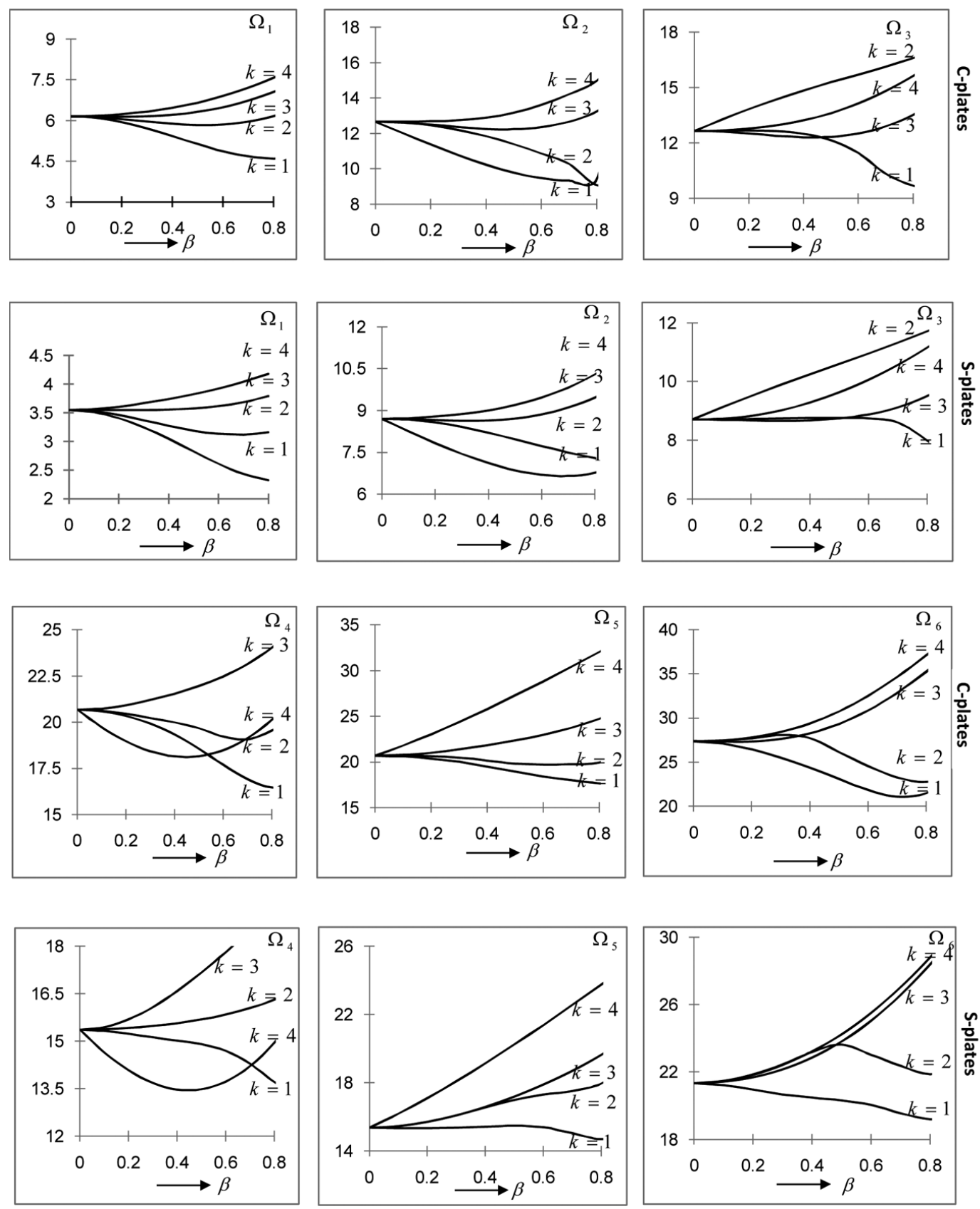

Fig. 4. Variation in $\Omega$ with $\beta$ for corresponding $\mathrm{C}$ and S- plates when $\alpha=0.5$ and $k=1,2,3$ and 4. 

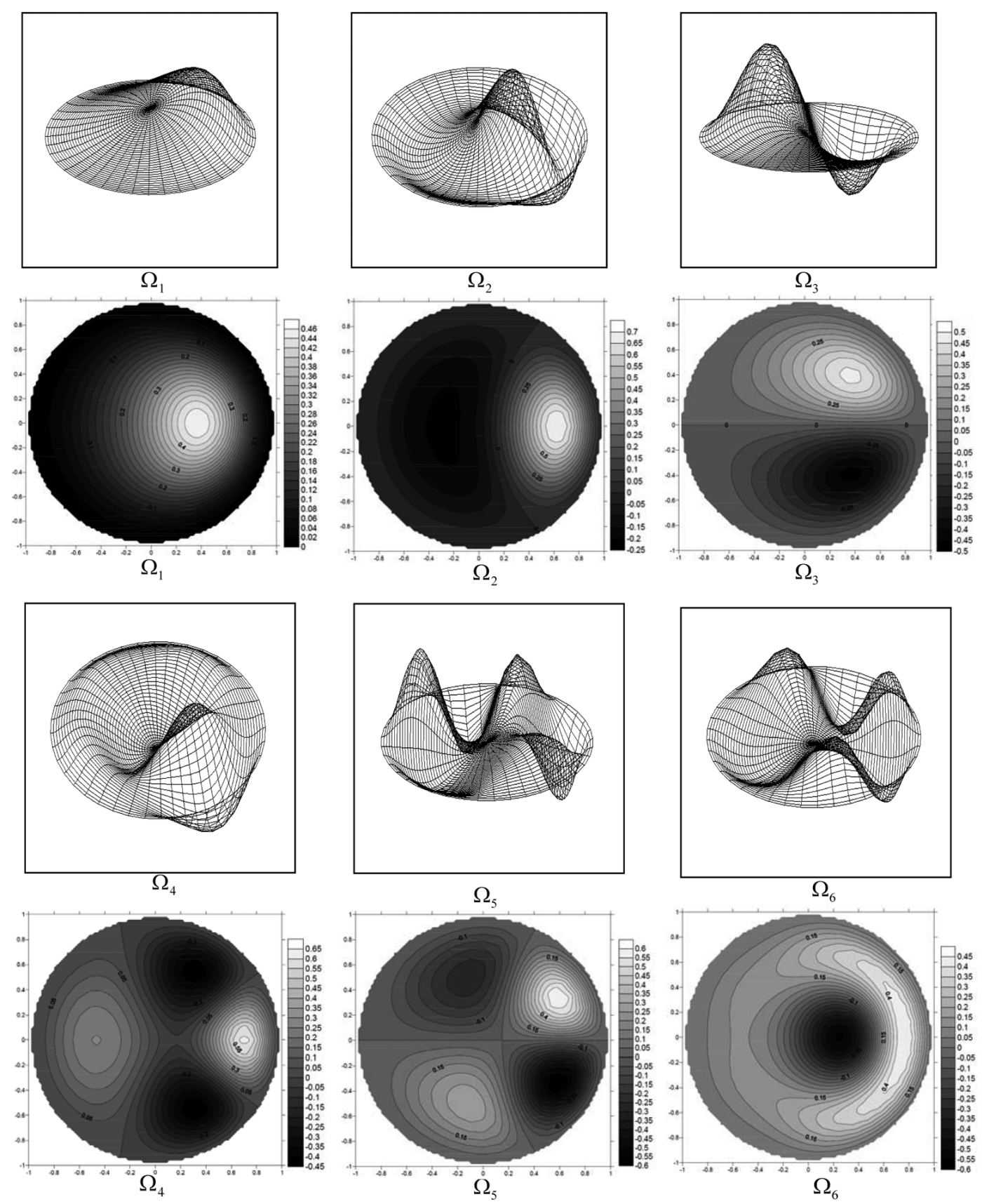

Fig. 5. First six normal modes and their contours for C-plates when $\alpha=\beta=0.5$ and $k=1$. 

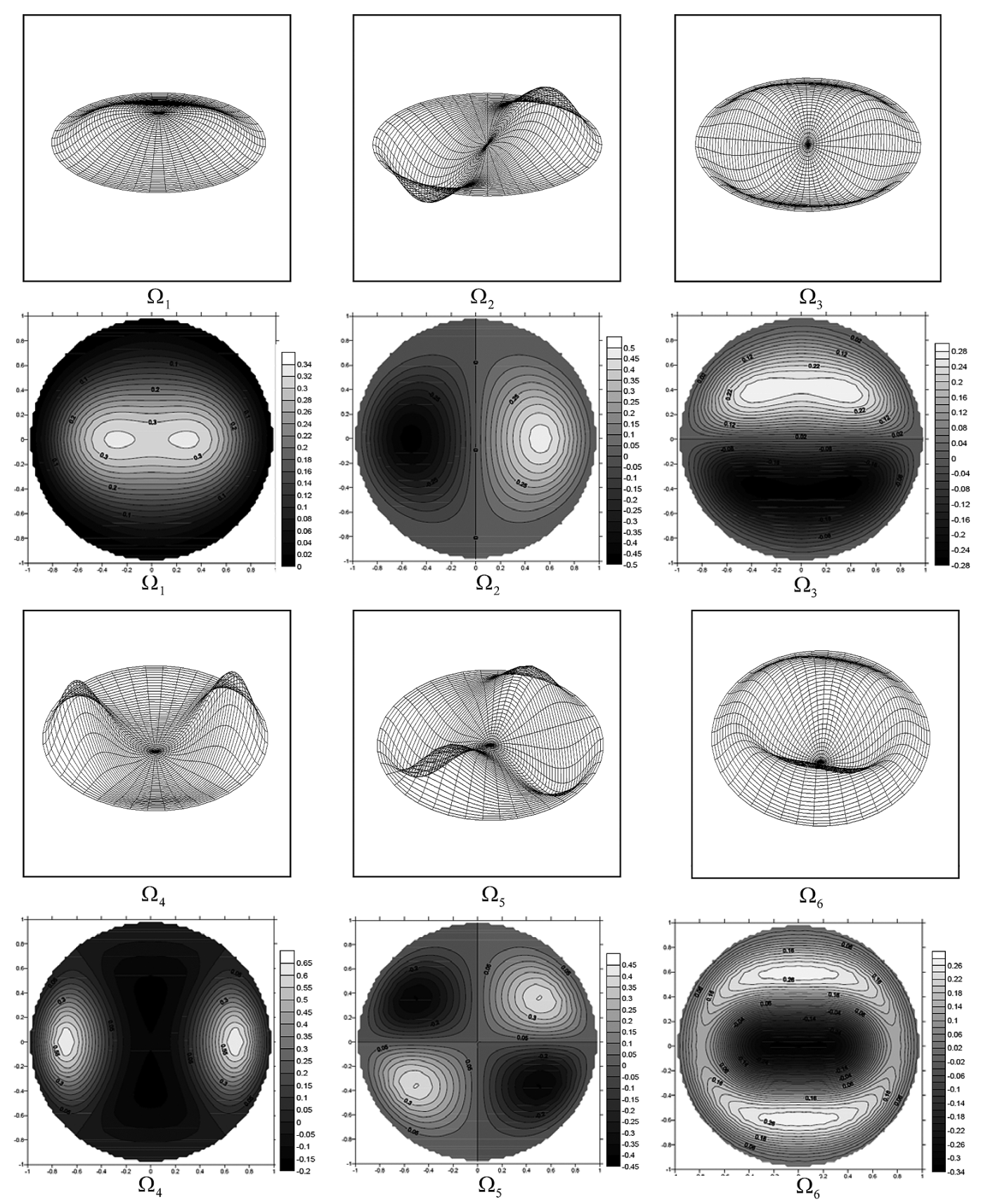

Fig. 6. First six normal modes and their contours for C-plates when $\alpha=\beta=0.5$ and $k=2$. 

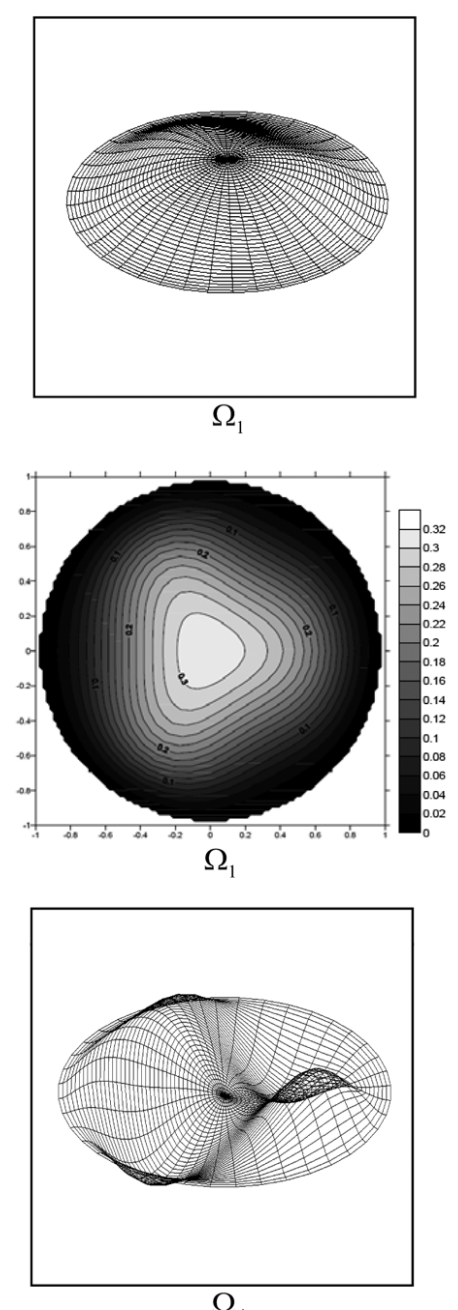

$\Omega_{4}$

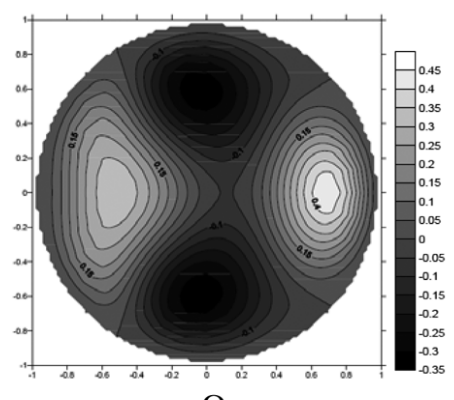

$\Omega_{4}$

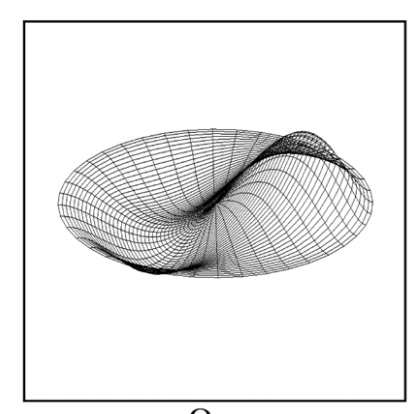

$\Omega_{2}$

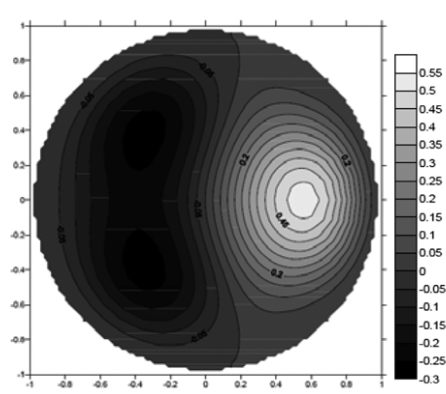

$\Omega_{2}$

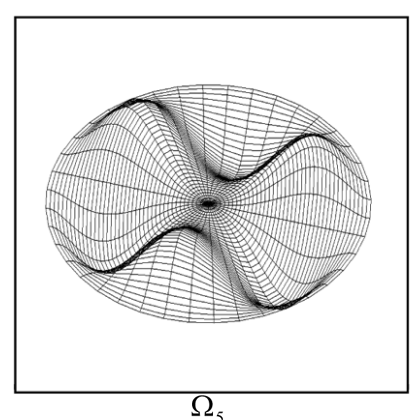

$\Omega_{5}$

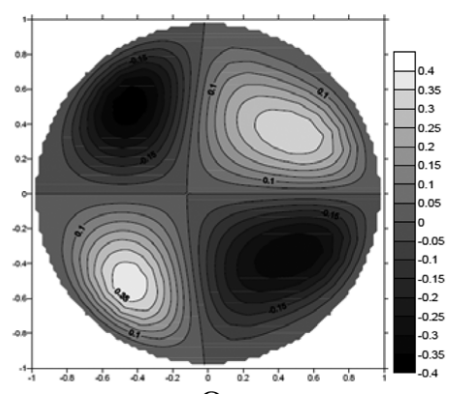

$\Omega_{5}$
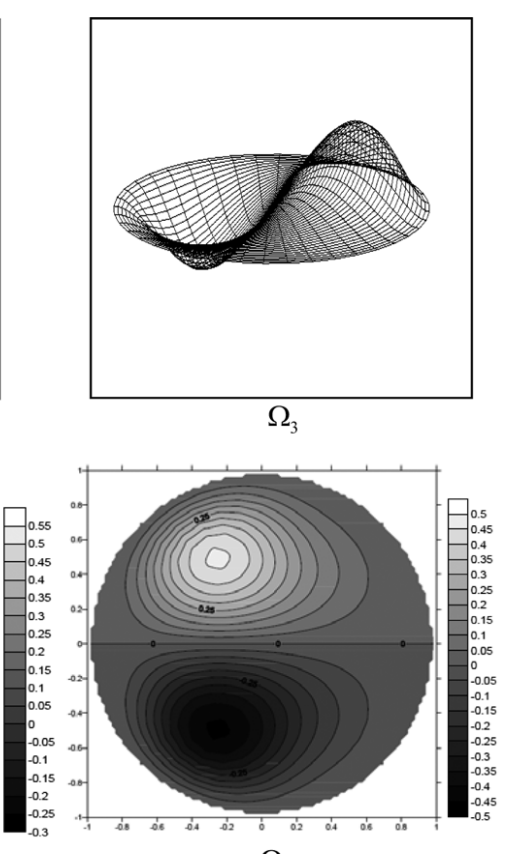

$\Omega_{3}$
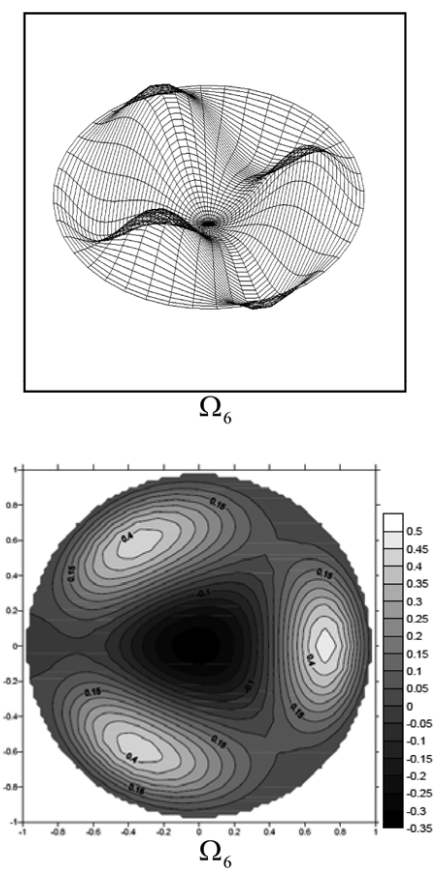

Fig. 7. First six normal modes and their contours for C-plates when $\alpha=\beta=0.5$ and $k=3$. 

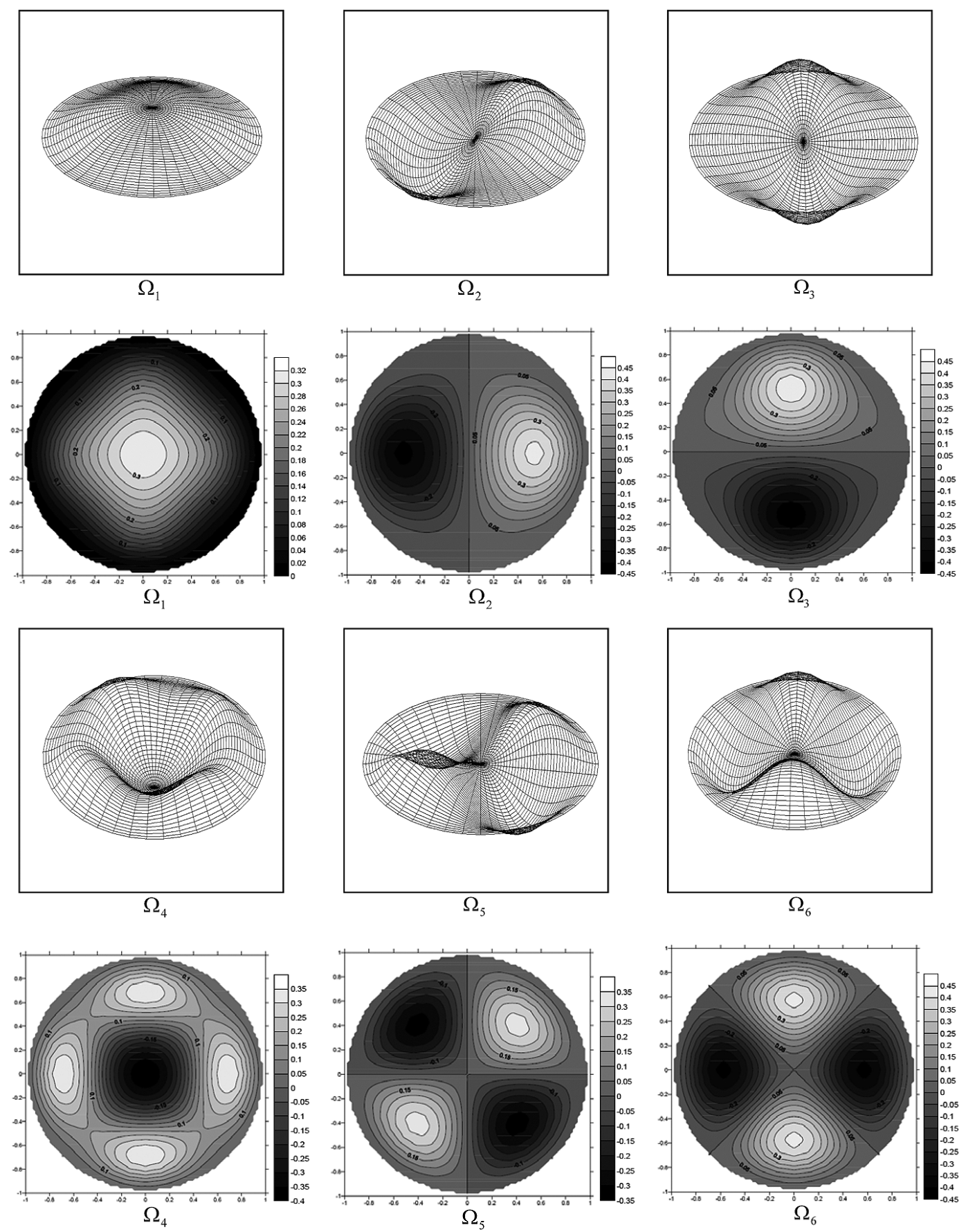

Fig. 8. First six normal modes and their contours for C-plates when $\alpha=\beta=0.5$ and $k=4$. 
Table 3

Comparison of $\Omega$ for isotropic circular plates of uniform thickness with simply supported edge when $\alpha=\beta=0$ and $e_{r}=1$

\begin{tabular}{lllll}
\hline Ref. & $\Omega_{1}$ & $\Omega_{2}$ & $\Omega_{3}$ & $\Omega_{4}$ \\
\hline$[24]$ & 4.93 & - & - & - \\
{$[25]$} & 4.935 & - & - & - \\
{$[26]$} & 4.94 & 29.72 & - & - \\
{$[27]$} & 4.977 & 29.72 & - & - \\
{$[28]$} & 4.935 & 29.720 & 74.24 & - \\
{$[2]$} & 4.9352 & 29.720 & 74.156 & - \\
{$[1]$} & 4.977 & 29.76 & 74.20 & 138.34 \\
Present & 4.9351 & 29.720 & 74.156 & 138.32 \\
\hline
\end{tabular}

Table 4

Comparison of $\Omega$ for isotropic circular plates of linearly varying thickness with simply supported

\begin{tabular}{|c|c|c|c|c|c|c|c|c|c|c|c|}
\hline \multirow{2}{*}{$\vec{\downarrow} \alpha$} & \multicolumn{4}{|c|}{$\Omega_{1}$} & \multicolumn{4}{|c|}{$\Omega_{2}$} & \multicolumn{3}{|c|}{$\Omega_{3}$} \\
\hline & [2] & [29] & [4] & Present & [2] & [29] & [4] & Present & [2] & [29] & Present \\
\hline 0.2 & - & - & 4.44 & 4.4345 & - & - & 26.54 & 26.462 & - & - & - \\
\hline 0.1 & 4.667 & 4.6637 & 4.71 & 4.7076 & 28.080 & 28.077 & 28.16 & 28.118 & 70.225 & 70.213 & 70.213 \\
\hline 0.0 & - & 4.9351 & 4.98 & 4.9351 & - & 29.720 & 29.77 & 29.720 & - & 74.156 & 74.156 \\
\hline-0.1 & 5.209 & 5.2061 & 5.25 & 5.2495 & 31.349 & 31.346 & 31.38 & 31.374 & 78.015 & 78.032 & 78.030 \\
\hline-0.2 & - & - & 5.52 & 5.5198 & - & - & 32.98 & 32.980 & - & - & - \\
\hline
\end{tabular}

Edge when $\beta=0, e_{r}=1.0, g_{r}=0.384, \nu_{r}=0.3$ and $K_{f}=0$.

Table 5

Comparison of $\Omega$ for $C$-plate when $\beta=0$

\begin{tabular}{rrrrrr}
\hline \multirow{2}{*}{$\Omega$} & \multicolumn{2}{c}{$\Omega_{1}$} & & \multicolumn{2}{c}{$\Omega_{2}$} \\
\cline { 2 - 3 } \cline { 5 - 6 } & \multicolumn{1}{c}{$[4]$} & Present & & {$[4]$} & Present \\
\hline 0.2 & 8.63 & 8.6020 & & 35.10 & 34.973 \\
0.1 & 9.44 & 9.4085 & & 37.47 & 37.438 \\
0.0 & 10.25 & 10.215 & & 39.81 & 39.921 \\
-0.1 & 11.05 & 11.024 & & 42.15 & 42.432 \\
-0.2 & 11.86 & 11.835 & & 44.49 & 44.977 \\
\hline
\end{tabular}

in a decrease in the stiffness of the plate. Also, when $\alpha$ is positive (i.e. larger mass concentrating at the center) makes the plate vibrates for a longer period or a lower frequency.

The variations of $\Omega$ for $\beta$ varying from -0.7 to 0.7 while $\alpha$ is kept constant (i.e. $\alpha=0.5$ ) are shown in Fig. 4 . It can be seen that in all the four cases $(k=1,2,3$ and 4$)$, when circumferential thickness $(1-\beta \cos k \theta)$ varies only and the radial thickness $(1-\alpha R)$ is kept constant, there is no fix pattern in frequencies. For the fundamental frequency $\Omega_{1}$ of both $\mathbf{C}$ - and $\mathbf{S}$-plates, it can be seen that the values remain almost constant for different $k$ when $\beta$ is small $(\beta \leqslant 0.15$ for $\mathbf{C}$-plate and $\beta \leqslant 0.1$ for $\mathbf{S}$-plate). Moreover, it is seen that the rate of increase of fundamental frequency with respect to $\mathrm{k}$ is faster for $\mathbf{S}$-plates in comparison with $\mathbf{C}$-plates for all $k$.

Three dimensional mode shapes and their corresponding contours of $\mathbf{C}$-plates for the first six normal modes of vibrations corresponding to $k=1,2,3,4$ are shown in Figs 5 to 8 when $\alpha=\beta=0.5, e_{r}=1.0$ and $\nu=0.3$. From the modes shown for a particular $k$, it is found that the boundary condition does not have significant influence on the mode shape when changes from clamped to simply-supported.

\section{Conclusion}

This paper deals with the free vibration of a class of plate having thickness variation in both radial and circumferential directions. Two dimensional boundary characteristic orthonormal polynomials are used in the Ritz method for the formulation of the eigenvalue problem. The accuracy and rate of convergence are shown. Comparison with results of special cases already reported in the literature shows good agreement for all the cases. It is found that 
the taper parameter for thickness variation in radial direction has more uniform influence on the changes in the magnitude of the frequencies in comparison with that in circumferential direction. Investigation of mode shapes shows that the clamped and simply-supported boundary condition has no significant influence.

\section{References}

[1] A.W. Leissa, Vibration of Plates, Acoustic Society of America (Originally published 1969 by Washington: Office of Technology Utilization, NASA SP-160), 1993.

[2] A.W. Leissa and Y. Narita, Natural frequencies of simply supported circular plates, Journal of Sound and Vibration 70 (1980), $221-229$.

[3] P.A.A. Laura and R.H. Gutierrez, Analysis of vibrating circular plates of non uniform thickness by the method of differential quadrature, Ocean Engineering 22 (1995), 97-100.

[4] P.A.A. Laura, R.H. Gutierrez, R.H. Carinicer and H.C. Sanzi, Free vibration of a solid circular plate of linearly varying thickness and attached to a Winkler foundation, Journal of Sound and Vibration 144(1) (1991), 149-162.

[5] D.V. Bambill and P.A.A. Laura, Comments on: Axisymmetric vibration of a circular plate with double linear variable thickness, Journal of Sound and Vibration 197(1) (1996), 131-135.

[6] S. Chakraverty, R.B. Bhat and I. Stiharu, Recent research on vibration of structures using boundary characteristic orthogonal polynomials in the Rayleigh- Ritz method, The Shock and Vibration Digest 31(3) (1999), 187-194.

[7] A.P. Gupta and N. Goyal, Forced axisymmetric response of linearly tapered circular plates, International Journal of Mechanical Sciences 36 (1994), 439-448.

[8] A.P. Gupta and N. Goyal, Forced asymmetric response of linearly tapered circular plates, Journal of Sound and Vibration 220(4) (1999), 641-657.

[9] C.S. Kim and S.M. Dickinson, On the lateral vibration of thin annular and circular composite plates subjected to certain complicated effects, Journal of Sound and Vibration 130(3) (1989), 363-377.

[10] D.Y. Chen, Axisymmetric vibration of circular and annular plates with arbitrary varying thickness, Journal of Sound and Vibration 206(1) (1997), 114-121.

[11] J. Wang, Generalized power series solutions of the vibration of classical circular plates with variable thickness, Journal of Sound and Vibration 202(4) (1997), 593-599.

[12] L.T.T. Hang, C.M. Wang and T.Y. Wu, Exact vibration results for stepped circular plates with free edges, International Journal of Mechanical Sciences 4 (2005), 1224-1248.

[13] W.H. Duan, C.M. Wang and C.Y. Wang, Modification of fundamental vibration of circular plates with free edges, Journal of Sound and Vibration 317(3-5) (2008), 709-715.

[14] N. Bhardwaj and A.P. Gupta, Axisymmetric vibrations of polar orthotropic circular plates of quadratically varying thickness resting on elastic foundation, International Journal of Structural Stability and Dynamics 5(3) (2005), 387-408.

[15] A.P. Gupta and N. Bhardwaj, Free vibration of polar orthotropic circular plates of quadratically varying thickness resting on elastic foundation, Journal of Applied Mathematical Modelling 29 (2005), 137-157.

[16] N. Bhardwaj and A.P. Gupta, Axisymmetric vibrations of polar orthotropic circular plates of quadratically varying thickness resting on elastic foundation, International Journal of Structural Stability and Dynamics 5(3) (2005), 387-408.

[17] N. Bhardwaj, A.P. Gupta and K.K. Choong, Vibration of orthotropic quarter elliptic plates with simply supported curved boundary and other complicated effects, Tamkang Journal of Science and Engineering 9(1) (2006), 1-18.

[18] N. Bhardwaj, A.P. Gupta and K.K. Choong, Free vibration of isotropic half elliptic plates of linearly varying thickness with clamped curved boundary, IIUM Engineering Journal Malaysia 7(2) (2006), 1-15.

[19] N. Bhardwaj, A.P. Gupta and K.K. Choong, Effect of elastic foundation on the vibration of orthotropic elliptic plates of varying thickness, MECCANICA International journal of the Italian Association of Theoretical and Applied Mechanics AIMETA, (40) (2007), 341-358.

[20] N. Bhardwaj, A.P. Gupta and K.K. Choong, Asymmetric vibration of polar orthotropic annular circular plates of quadratically varying thickness with same boundary conditions, Shock and Vibration (15) (2008), 599-617.

[21] A.H. Ansari and U.S. Gupta, Forced axi-symmetric response of polar orthotropic tapered circular plates resting on elastic foundation, Indian Journal of Pure and Applied Mathematics 37(4) (2006), 209-221.

[22] O. Civalek and B. Ozturk, Discrete singular convolution method for free vibration analysis of tapered rectangular plates, Advances in Vibration Engineering 7(3) (2008), 261-274.

[23] O. Civalek, Fundamental frequency of isotropic and orthotropic rectangular plates with linearly varying thickness by discrete singular convolution method, Applied Mathematical Modeling 33(10) (2009), 3825-3835.

[24] R. Gelos, G.M. Ficcadenti, R.O. Grossi and P.A.A. Laura, Vibrations of circular plates with variable profile, Journal of the Acoustical Society of America 69(5) (1981), 1326-1329.

[25] L.E. Luisoni, P.A.A. Laura and R.O. Grossi, Antisymmetric modes of vibration of a circular plate elastically restrained against rotation and of linearly varying thickness, Journal of Sound and Vibration 55 (1977), 461-466.

[26] R.O. Wah, Vibration of circular plates, Journal of the Acoustical Soc of America 34(3) (1962), $275-281$.

[27] V.S. Gontkevich, Natural Vibrations of Plates and Shells, (A.P. Filippov, editor), Kiev: Nauk Dumka, 1964.

[28] D. Avalos, P.A.A. Laura and A.M. Bianchi, Analytical and experimental investigation on vibrating circular plates with stepped thickness over a concentric circular region, Journal of Acoustic Society of America 82 (1987), 13-16.

[29] B. Singh and B. Saxena, Axisymmetric vibration of circular plate with double variable thickness, Journal of Sound and Vibration 179 (1995), 879-897. 

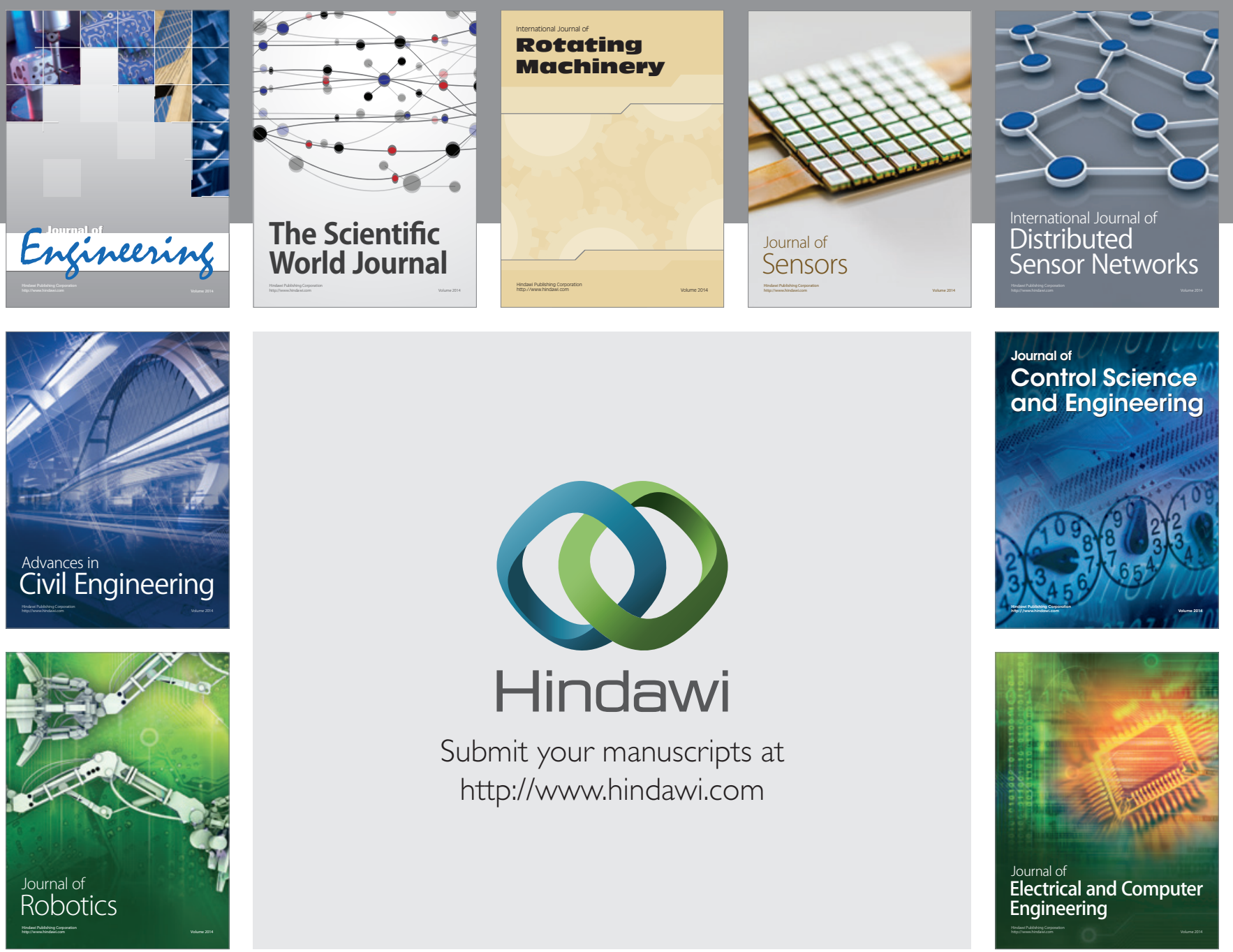

Submit your manuscripts at

http://www.hindawi.com
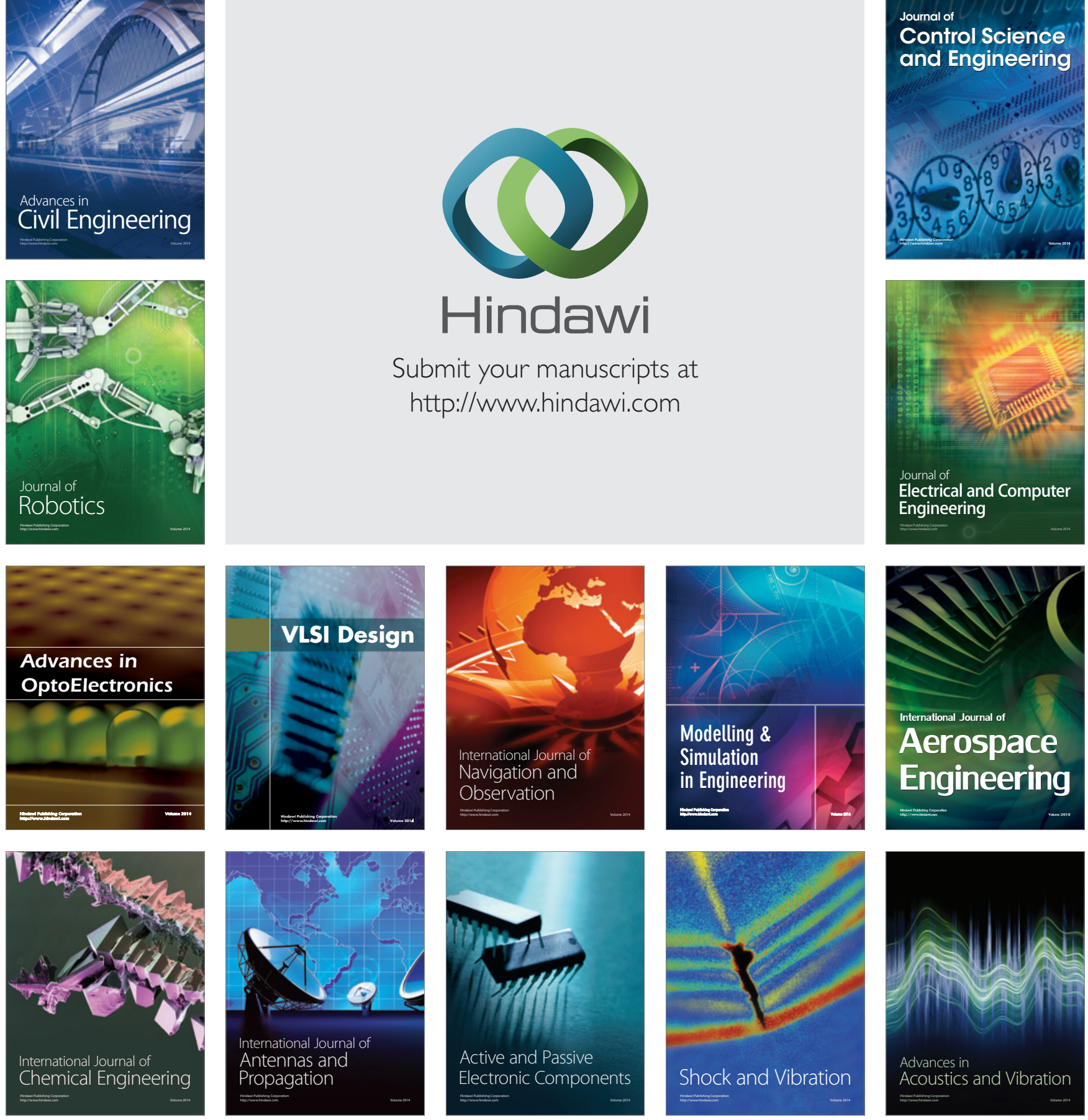